\title{
Water assistance: also for sigmoidoscopy?
}

\section{(웅 $\circledast$}

\author{
Author \\ Felix W Leung ${ }^{1,2}$ \\ Institutions \\ 1 Sepulveda Ambulatory Care Center, Veterans Affairs \\ Greater Los Angeles Healthcare System, North Hills, \\ California, United States \\ 2 David Geffen School of Medicine at University of \\ California at Los Angeles, Los Angeles, California, United \\ States
}

Bibliography

DOI https://doi.org/10.1055/a-0959-6057 |

Endoscopy International Open 2020; 08: E3-E5

\author{
(c) Georg Thieme Verlag KG Stuttgart · New York \\ elSSN 2196-9736 \\ Corresponding author \\ Felix W. Leung, MD, 111G, Gastroenterology Sepulveda \\ Ambulatory Care Center, Veterans Affairs Greater Los \\ Angeles Healthcare System, 16111 Plummer Street, North \\ Hill, CA \\ Fax: $+1-8188959516$ \\ felix.leung@va.gov
}

In this issue of Endoscopy International Open, the protocol for an ongoing randomized controlled trial (RCT) is described [1]. The primary objective is to ascertain if procedural pain, assessed post-procedure and prior to discharge, is reduced in water-assisted sigmoidoscopy (WAS) compared to carbon dioxide $\left(\mathrm{CO}_{2}\right)$ insufflation in people undergoing unsedated flexible sigmoidoscopy as part of the United Kingdom Bowel Scope Screening Program (BSSP). In the BSSP, an enema is given, colonoscope used, and Entonox (nitrous oxide) readily available as needed. Recognition that water instead of gas can potentially reduce insertion pain based on published reports on water-aided colonoscopy represents an important milestone in the history of water-assisted examination of the colon. This is a welldesigned trial which shows considerable promise in demonstrating a less painful method for people undergoing screening sigmoidoscopy. This editorial focuses on discussions of the timing of pain assessment and insertion technique.

Recording of pain data by a blinded research assistant immediately after examination of the colon is a sound experimental design to minimize observer bias. Pain during insertion, however, is a clinically relevant parameter as excessive insertion pain mandates discontinuation, or need to add Entonox. Therefore, real-time maximum insertion pain more accurately reflects the importance of pain reduction and is a pertinent measure to track [2-4] in addition to the current primary outcome.

Recognition of the need to reduce pain related to gas insufflation is critical to development of the protocol. The investigators should be commended for addressing the issue with this RCT using water-assisted technique. At a risk of stating the obvious, there are two main approaches to water assistance for examination of the colon, namely, water immersion (WI) and water exchange (WE) [5]. In the English-language literature, WI dates back to 1984 [6]. Water was administered into the sigmoid colon deformed by diverticulosis to facilitate passage and the infused water was removed during withdrawal. The practice is simple and easy to adopt. The approach was extended to the entire colon as WE to facilitate completion of colonoscopy in unsedated veterans in the United States. WE emphasizes removal of the infused water (and residual debris) during insertion to minimize distension and optimize reduction of realtime maximum insertion pain [2]. The serendipitous consequences of salvage cleaning during insertion and reduced multi-tasking distractions due to cleaning activities during withdrawal [7] are likely instrumental in the increase in adenoma detection when WE is used [8-10]. The key to success of WE appears to be near-complete suction removal of the infused water (along with residual debris) during insertion [11].

The WAS technique (water infusion based on endoscopist discretion, suction of infused water as needed or as per usual practice during withdrawal) [1] appears to resemble WI ( $\triangleright$ Table 1). Data in the literature would suggest that WAS may need the addition of standardized water suction during insertion to achieve effectiveness as optimal as WE in minimizing insertion pain or increasing adenoma detection. To maximize the benefits of water assistance, it is prudent to consider use of WE for the proposed study, i.e. with near-complete suction removal of the infused water during insertion, and not just "as needed." The history of water-aided colonoscopy showed that the change from WI to WE $[3,4]$ reaped important benefits of significant further reduction of insertion pain.

When WE is indeed adopted for flexible sigmoidoscopy, there is the potential to attempt extended flexible sigmoidoscopy [12], especially when a colonoscope is already being 
- Table 1 Differences between water exchange and water immersion suggest water-assisted sigmoidoscopy is similar to water immersion.

\begin{tabular}{|l|l|l|l|}
\hline Characteristics & WE (colonoscopy) & WI (colonoscopy) & WAS (sigmoidoscopy) \\
\hline Maneuvers & Standardized & $\begin{array}{l}\text { Based on discretion of endos- } \\
\text { copist }\end{array}$ & Based on discretion of endoscopist \\
\hline Gas pump turned off & Before insertion & Before or after & After \\
\hline Use of blast of gas & No & Allowed & Allowed \\
\hline Suction of luminal gas & Yes & Optional & Yes \\
\hline $\begin{array}{l}\text { Suction of infused water and resi- } \\
\text { dual debris }\end{array}$ & $\begin{array}{l}\text { Near-complete removal during } \\
\text { insertion }\end{array}$ & $\begin{array}{l}\text { Removal mainly during with- } \\
\text { drawal }\end{array}$ & $\begin{array}{l}\text { As needed and as per standard practice } \\
\text { during withdrawal }\end{array}$ \\
\hline Salvage cleaning during insertion & Integral component & Optional & As needed \\
\hline Cleaning during withdrawal & Minimal after insertion cleaning & Yes & Yes \\
\hline Insertion pain reduction & $\begin{array}{l}\text { Better than WI, Al or } \mathrm{CO}_{2} \text { insuf- } \\
\text { flation }\end{array}$ & $\begin{array}{l}\text { Better than Al or CO2 insuf- } \\
\text { flation }\end{array}$ & $\begin{array}{l}\text { Primary outcome of current RCT, likely } \\
\text { comparable to WI }\end{array}$ \\
\hline Al, air insufflation; $\mathrm{CO}_{2}$, carbon dioxide; $\mathrm{RCT}$ randomized controlled trial; WAS, water-assisted sigmoidoscopy; WE, water exchange; WI, water immersion.
\end{tabular}

used in the BSSP. The only significant change is for the patient to undertake an oral bowel preparation to clean the entire coIon. To the fullest extent of intubation, a WE-assisted extended flexible sigmoidoscopy is equivalent to an unsedated colonoscopy. The increased coverage of and discovery of polyps in the proximal colon without the expense and burden of sedation and return visit to remove polyps beyond the reach of the sigmoidoscope can be substantial. This would be a reasonable next step for investigators in this RCT to consider.

On a lighter note, the current RCT confirms that the investigators appreciate the disadvantages of gas insufflation for insertion. On the other hand, WE has distinct advantages. The investigators could consider trying to avoid using any insufflation at all and achieve suction salvage cleaning during insertion (should be easy now that the skills in water-assisted technique have been acquired). The full benefits of further decrease in pain and increase in adenoma detection, and possibly a greater chance to complete a higher proportion of unsedated colonoscopies than using the current technique, could be achieved. It is reasonable to experiment and have fun. Performing WE is neither boring nor excessively time-consuming in a reasonably well-prepared colon. In addition to less intense insertion pain for the patient, the annoying task of cleaning up during withdrawal can be avoided and the hunt for lesions will become more enjoyable for the colonoscopist.

\section{Acknowledgements}

This editorial is supported in part by a VA Merit Research Grant and a Clinical Research Grant provided by the American Society for Gastrointestinal Endoscopy

\section{Competing interests}

None

References

[1] Beintaris I, Esmaily S, Saunders BP et al. The WASh Trial: Water-assisted Sigmoidoscopy in the United Kingdom Bowel Scope Screening Programme: study protocol for a randomized multicentre trial. Endosc Int Open 2019; 07: E1574-E1582

[2] Leung FW, Harker JO, Jackson G et al. A proof-of-principle, prospective, randomized controlled trial (RCT) demonstrating improved outcomes in scheduled unsedated colonoscopy by the water method. Gastrointest Endosc 2010; 72: 693-700

[3] Hsieh YH, Koo M, Leung FW. A patient-blinded randomized, controlled trial (RCT) comparing air insufflation (Al), water immersion (WI) and water exchange (WE) during minimally sedated colonoscopy. Am J Gastroenterol 2014; 109: 1390 - 1400

[4] Cadoni S, Sanna S, Gallittu P et al. A randomized controlled trial comparing real-time insertion pain during colonoscopy confirmed water exchange to be superior to water immersion in enhancing patient comfort. Gastrointest Endosc 2015; 81: 557- 566

[5] Leung FW, Amato A, Ell C et al. Water-aided colonoscopy: a systematic review. Gastrointest Endosc 2012; 76: 657-666

[6] Falchuk ZM, Griffin PH. A technique to facilitate colonoscopy in areas of severe diverticular disease. N Engl J Med 1984; 310: 598

[7] Hsieh YH, Koo M, Tseng CW et al. Reduction of multitasking distractions underlies the higher adenoma detection rate of water exchange compared to air insufflation - blinded analysis of withdrawal phase videos. United European Gastroenterol 2019; 7: 230-238

[8] Jia H, Pan Y, Guo X et al. Water exchange method significantly improves adenoma detection rate: a multicenter, randomized controlled trial. Am J Gastroenterol 2017; 112: 568 - 576

[9] Hsieh YH, Tseng CW, Hu CT et al. Prospective multicenter randomized controlled trial demonstrating water exchange (WE), but not water immersion (WI), significantly increases adenoma detection compared with air insufflation (AI) even in propofol sedated patients. Gastrointest Endosc 2017; 86: $192-201$ 
[10] Cadoni S, Falt P, Rondonotti E et al. Water exchange for screening colonoscopy increases adenoma detection rate: a multicenter, doubleblinded, randomized controlled trial. Endoscopy 2017; 49: 456- 467

[11] Leung FW, Jia H. Water infusion without near-complete removal during insertion by any other name is still water immersion (WI). Gastrointest Endosc 2019; 89: 599-601
[12] Bak AW, Perini R, Schroeder T et al. Experience with water-aided colonoscopy in a Canadian community population. Journal Interventional Gastroenterology 2013; 3: 49-52 